\title{
Comparison of different methods of splenic hilar lymph node dissection for advanced upper- and/or middle-third gastric cancer
}

\author{
Xin $\mathrm{Ji}^{\dagger}$, Tao Fu ${ }^{\dagger}$, Zhao-De Bu, Ji Zhang, Xiao-Jiang Wu, Xiang-Long Zong, Zi-Yu Jia, Biao Fan, Yi-Nan Zhang
} and Jia-Fu Ji ${ }^{*}$

\begin{abstract}
Background: Surgery for advanced gastric cancer (AGC) often includes dissection of splenic hilar lymph nodes (SHLNs). This study compared the safety and effectiveness of different approaches to SHLN dissection for upperand/or middle-third AGC.

Methods: We retrospectively compared and analyzed clinicopathologic and follow-up data from a prospectively collected database at the Peking University Cancer Hospital. Patients were divided into three groups: in situ spleenpreserved, ex situ spleen-preserved and splenectomy.

Results: We analyzed 217 patients with upper- and/or middle-third AGC who underwent R0 total or proximal gastrectomy with splenic hilar lymphadenectomy from January 2006 to December 2011, of whom 15.2 \% (33/ 217) had metastatic SHLNs, and from whom $11.4 \%$ (53/466) of the dissected SHLNs were metastatic. The number of harvested SHLNs per patient was higher in the ex situ group than in the in situ group $(P=0.017)$. Length of postoperative hospital stay was longer in the splenectomy group than in the in situ group $(P=0.002)$ or the ex situ group $(P<0.001)$. The splenectomy group also lost more blood volume $(P=0.007)$ and had a higher postoperative complication rate $(P=0.005)$ than the ex situ group. Kaplan-Meier (log rank test) analysis showed significant survival differences among the three groups $(P=0.018)$. Multivariate analysis showed operation duration $(P=0.043)$, blood loss volume $(P=0.046)$, neoadjuvant chemotherapy $(P=0.005)$, and $\mathrm{N}$ stage $(P<0.001)$ were independent prognostic factors for survival.

Conclusions: The ex situ procedure was more effective for SHLN dissection than the in situ procedure without sacrificing safety, whereas splenectomy was not more effective, and was less safe. The SHLN dissection method was not an independent risk factor for survival in this study.
\end{abstract}

Keywords: Advanced gastric cancer, Splenic hilar lymph node dissection, Splenic preservation, Splenectomy

\section{Background}

The estimated incidence and mortality of gastric cancer in 2013 were 984,000 and 841,000 worldwide, respectively $[1,2]$. Globally, gastric cancer is the fifth most common cancer and the second most common cause of cancer death. More than $70 \%$ of these cases occur in developing countries, with half arising in Eastern Asia

\footnotetext{
*Correspondence: jijiafu_pku@163.com

${ }^{\dagger}$ Equal contributors

Department of Gastrointestinal Surgery, Key laboratory of Carcinogenesis and Translational Research (Ministry of Education), Peking University Cancer Hospital \& Institute, Haidian District Fucheng Road No. 52, Beijing 100142, China
}

(mainly Korea, Japan, and China). Surgery is the primary treatment for gastric cancer, with D2 lymphadenectomy widely accepted for advanced gastric cancer (AGC) in both Eastern and Western countries [3-5].

The incidence of upper- and/or middle-third gastric cancer has steadily increased, especially in Asia [6]. According to the 2010 Japanese gastric cancer treatment guideline (ver. 3) published by the Japanese Gastric Cancer Association, the extent of systematic lymphadenectomy depends on the type of gastrectomy [7]. The lymph node stations surrounding the stomach have been precisely defined by the Japanese Gastric Cancer 
Association (Table 1 and Fig. 1). To achieve sufficient negative proximal margins, most patients with upperand/or middle-third AGC require total gastrectomies with D2 lymphadenectomies that include the splenic hilar lymph nodes (SHLNs; No. 10 lymph nodes) [8].

Reportedly, 7.3-26 \% of SHLNs in upper- and/or middle-third AGC are metastatic [9-12]. Prophylactic splenectomy, in situ and ex situ spleen-preserving lymphadenectomies have been the most common dissection approaches for SHLNs. Prophylactic splenectomy was a common procedure for D2 dissection until the results of the Japanese Clinical Oncology Group (JCOG) 0110 study that showed a non-inferiority of spleen preservation compared with splenectomy in terms of overall survival $[13,14]$. Nonetheless, as the JCOG 0110 study included only tumors from the lesser curvature, the approach for patients with tumors at the greater curvature is still in doubt.

Two main operative procedures for SHLN dissection spare the spleen. Ex situ and in situ dissection are defined depending on whether the pancreas and spleen are treated within the peritoneal cavity or not. The in situ dissection approach is more difficult as the SHLN dissection is implemented in a narrow and small space,

Table 1 Regional lymph nodes for gastric cancer

\begin{tabular}{|c|c|}
\hline No & Definition \\
\hline 1 & Right paracardial LNs \\
\hline 2 & Left paracardial LNs \\
\hline $3 a$ & Lesser curvature LNs along the branches of the left gastric artery \\
\hline $3 b$ & $\begin{array}{l}\text { Lesser curvature LNs along the } 2 \text { nd branch and distal part of the } \\
\text { right gastric artery }\end{array}$ \\
\hline 4 sa & Left greater curvature LNs along the short gastric arteries \\
\hline $4 s b$ & Left greater curvature LNs along the left gastroepiploic artery \\
\hline $4 d$ & $\begin{array}{l}\text { Left greater curvature LNs along the } 2 \text { nd branch and distal part } \\
\text { of the right gastroepiploic artery }\end{array}$ \\
\hline 5 & $\begin{array}{l}\text { Suprapyloric LNs along the 1st branch and proximal part of the } \\
\text { right artery }\end{array}$ \\
\hline 6 & $\begin{array}{l}\text { Infrapyloric LNs along the 1st branch and proximal part of the } \\
\text { right gastroepiploic artery }\end{array}$ \\
\hline 7 & $\begin{array}{l}\text { LNs along the trunk of left gastric artery between its root and } \\
\text { the origin of tis ascending branch }\end{array}$ \\
\hline $8 \mathrm{a}$ & Anterosuperior LNs along the common hepatic artery \\
\hline $8 p$ & Posterior LNs along the common hepatic artery \\
\hline 9 & Celiac artery LNs \\
\hline 10 & Splenic hilar LNs \\
\hline $11 p$ & Proximal splenic artery LNs \\
\hline $11 d$ & Distal splenic artery LNs \\
\hline $12 \mathrm{a}$ & Hepatoduodenal ligaments LNs along the proper hepatic artery \\
\hline $12 \mathrm{p}$ & Hepatoduodenal ligaments LNs along the portal vein \\
\hline $12 b$ & Hepatoduodenal ligaments LNs along the bile duct \\
\hline
\end{tabular}

LNs lymph node

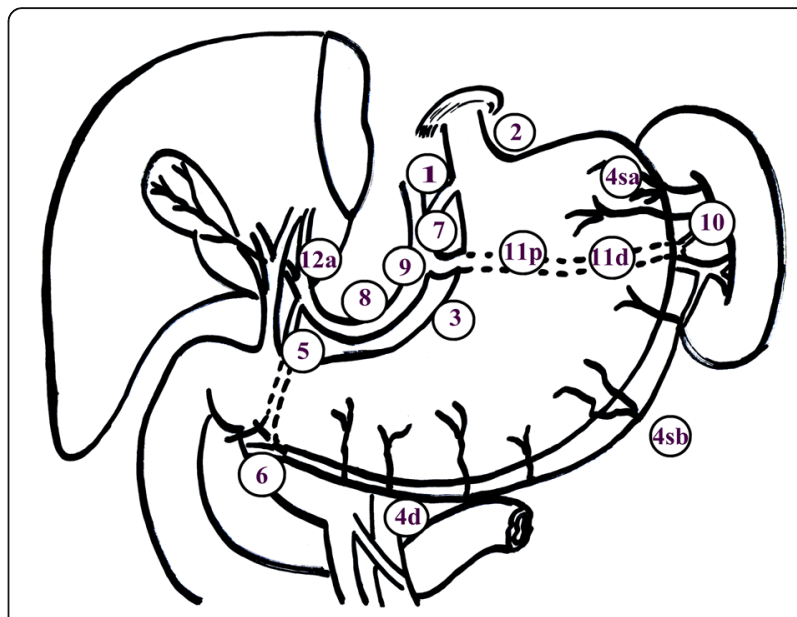

Fig. 1 Definition of lymph node stations of gastric cancer. The lymph nodes of stomach are defined and given station numbers. Lymph node stations1-7, 8a, 9, 10, 11p, 11d and 12a are included in the D2 dissection for locally advanced upper and/or middle third gastric cancer

and can thus lead to bleeding; however, it avoids moving the pancreas and spleen and shortens surgical time. In contrast, ex situ dissection is performed under direct vision, which provides a better exposure, and is thus less difficult.

To our knowledge, no previous study has directly compared the effectiveness and safety of these three approaches. We therefore investigated which of these three dissection approaches was better for patients with upper- and/or middle-third AGC.

\section{Methods}

Patients

This study was performed after approval by the Ethics Committee of Peking University Cancer Hospital. Informed consent was obtained from each patient. We retrospectively collected clinical and pathological data from a prospectively collected database at the Peking University Cancer Hospital. We included 217 patients with upper- and/or middle-third AGC who had undergone R0 total or proximal gastrectomy with SHLN dissection from January 2006 to December 2011. Their primary diagnoses were confirmed by endoscopic biopsies analysis. Clinical staging was mainly confirmed by ultrasound endoscopy, chest, abdominal and pelvic computed tomography scans, and laparoscopic exploration. Patients with other types of tumors, such as gastrointestinal stromal tumor or lymphoma, were excluded.

\section{Surgical procedure}

All the enrolled patients underwent laparoscopic exploration to exclude distant metastatic disease. After that, all the patients received $\mathrm{R} 0$ resection with total or proximal 
gastrectomy and SHLN dissection. The lymph node dissection scope was mainly D2/D2+, according to the definition in the Japanese gastric cancer treatment guidelines [7]. The approach of SHLN dissection was at the discretion of the surgeon during the operation.

In the splenectomy group, splenectomy was performed with full mobilization of the distal pancreas and spleen. Lymph nodes along the splenic artery were completely dissected. The splenic artery was usually ligated and divided 5-6 $\mathrm{cm}$ away from its origin. The spleen and lymph nodes at the hilum of the spleen were removed, with the pancreas preserved.

In the in situ spleen-preserved group, the spleen and the pancreas were not mobilized from the retroperitoneum. Lymph nodes along the splenic artery were dissected. All the soft tissues at the splenic hilum were removed as cautiously as possible.

In the ex situ spleen-preserved group, splenic hilar lymphadenectomy was performed with full mobilization of the distal pancreas and spleen. The spleen was moved outside the peritoneal cavity. Lymph nodes along the splenic artery and at the splenic hilum were completely dissected, with the pancreas and spleen preserved, and then replaced into the peritoneal cavity.

After the surgery, the patients stayed in hospital to get recovery. Before they left the hospital, the discharge criteria must be all fulfilled. The discharge criteria included: absence of subjective complaints, tolerance of solid oral intake, return of bowel function, absence of intravenous fluids/medications, adequate mobility of daily living and self-care (eg, go to toilet, dress, shower, etc.), adequate pain control on oral analgesia only, adequate wound condition, removal of drainage tube, absence of infectious complications, absence of postoperative complications, absence of abnormal physical signs or laboratory test (eg, pulse, body temperature, white blood cell count, serum hemoglobin, etc.), acceptance of discharge, adequate home/social condition.

\section{Clinicopathologic parameters}

The clinicopathological data collected from the database included age, sex, body mass index (BMI), neoadjuvant chemotherapy (NACT) regimens, tumor location, tumor size, presence of multi-tumor, range of gastrectomy, degree of lymph node dissection (LND), SHLN dissection procedure, tumor differentiation, lymphovascular invasion (LVI), depth of tumor invasion, number of harvested and metastatic lymph nodes, postoperative complications, mortality, length of postoperative hospital stay, operation duration, blood loss volume, and survival outcomes. Terminology used to describe the clinicopathologic parameters was based on the Japanese Gastric Cancer Association classification of gastric carcinoma [8].

\section{Follow-up}

Follow-up was conducted mainly through telephone interviews, E-mail communication, or outpatient reviews. As of April 26, 2016, the percentage of follow-up was $96.7 \%(210 / 217)$.

\section{Statistical analysis}

All statistical analysis was performed through IBM SPSS Statistics 20.0 software (SPSS Inc., Armonk, NY). For quantitative variables, normal distribution was tested first. Variables of normal distribution were expressed as means \pm standard deviation, and tested by analysis of variance among the three groups. If not, the variables were expressed as medians with ranges, and compared by Kruskal-Wallis non-parametric test. For categorical data, the chi-squared test or Fisher's exact test was performed. Kaplan-Meier estimation and the log-rank tests were used to calculate survival. In the pairwise comparisons, the original calculated $\mathrm{P}$ value and the Bonferronicorrected threshold were listed. If the $\mathrm{P}$ value was less than this Bonferroni-corrected threshold, then the comparison was considered to be statistically significant. Cox proportional hazards regression model was used to confirm independent prognostic factors through univariate and multivariate analysis. Except in the pairwise comparison, $P<0.05$ (two-sided) was considered significant in the statistical analysis.

\section{Results}

\section{Clinicopathologic parameters}

We analyzed 217 patients in this retrospective study, who were divided into three groups: in situ $(n=68)$, ex situ $(n=118)$, and splenectomy $(n=31)$. Some of the patients in the splenectomy group had intended to undergo in situ or ex situ approach after abdominal exploration, but encountered unintended splenic injury resulting in splenectomy. Of all the thirty-one patients in the splenectomy group, two patients underwent conversion from in situ approach to splenectomy, and three patients underwent conversion from ex situ approach to splenectomy. The rates of conversion from in situ and ex situ procedures to splenectomy were $2.86 \%(2 / 70)$ and $2.48 \%$ (3/121), respectively. All of their clinicopathologic factors except the number of patients who received NACT and the range of gastrectomy were comparable among the three groups; however, lower percentages of the in situ group underwent $\mathrm{NACT}$ and total gastrectomies than the ex situ and splenectomy groups (Table 2).

\section{Splenic hilar lymphadenectomy}

All 217 patients in our study underwent SHLN dissection, and all of the dissected lymph nodes were confirmed by pathological examination. Of the 217 patients, $33(15.2 \%)$ were found to have metastatic SHLNs, 
Table 2 Patients' clinicopathologic parameters

\begin{tabular}{|c|c|c|c|c|}
\hline & $\begin{array}{l}\text { In situ } \\
(n=68), n(\%)\end{array}$ & $\begin{array}{l}\text { Ex situ } \\
(n=118), n(\%)\end{array}$ & $\begin{array}{l}\text { Splenectomy } \\
(n=31), n(\%)\end{array}$ & Pvalue \\
\hline Gender & & & & 0.238 \\
\hline Male & $47(69.1)$ & $91(77.1)$ & $26(83.9)$ & \\
\hline Female & 21(30.9) & $27(22.9)$ & $5(16.1)$ & \\
\hline Age & & & & 1.000 \\
\hline$<60$ & $36(52.9)$ & 63(53.4) & $17(54.8)$ & \\
\hline$\geq 60$ & $32(47.1)$ & $55(46.6)$ & $14(45.2)$ & \\
\hline BMI & & & & 0.716 \\
\hline$<19$ & $5(7.4)$ & $10(8.5)$ & $1(3.2)$ & \\
\hline$\sim<25$ & $46(67.6)$ & $83(70.3)$ & $22(71.0)$ & \\
\hline$\sim<30$ & $17(25)$ & $22(18.6)$ & $7(22.6)$ & \\
\hline$\geq 30$ & $0(0)$ & $3(2.5)$ & $1(3.2)$ & \\
\hline NACT & & & & 0.008 \\
\hline No & $42(61.8)$ & $48(40.7)$ & $11(35.5)$ & \\
\hline Yes & $26(38.2)$ & $70(59.3)$ & $20(64.5)$ & \\
\hline Degree of LND & & & & 0.090 \\
\hline $\mathrm{D} 1+$ & $5(7.4)$ & $7(5.9)$ & $1(3.2)$ & \\
\hline D2 & $58(85.3)$ & $84(71.2)$ & $23(74.2)$ & \\
\hline $\mathrm{D} 2+$ & $5(7.4)$ & $27(22.9)$ & $7(22.6)$ & \\
\hline Gastrectomy & & & & 0.033 \\
\hline Proximal & $28(41.2)$ & $34(28.8)$ & $5(16.1)$ & \\
\hline Total & $40(58.8)$ & $84(71.2)$ & $26(83.9)$ & \\
\hline Differentiation & & & & 0.115 \\
\hline Well & $1(1.5)$ & $9(7.6)$ & $5(16.1)$ & \\
\hline Moderate & $31(45.6)$ & $53(44.1)$ & $12(38.7)$ & \\
\hline Poor & $36(52.9)$ & $57(48.3)$ & $14(45.2)$ & \\
\hline LVI & & & & 0.060 \\
\hline No & 26(38.8) & $66(55.9)$ & $12(41.4)$ & \\
\hline Yes & $41(61.2)$ & $52(44.1)$ & $17(58.6)$ & \\
\hline Location & & & & 0.648 \\
\hline EGJ & $35(51.5)$ & $63(53.4)$ & $12(38.7)$ & \\
\hline U/UM & $7(10.3)$ & $10(8.5)$ & $6(19.4)$ & \\
\hline $\mathrm{M} / \mathrm{MU}$ & 24(35.3) & $43(36.4)$ & $12(38.7)$ & \\
\hline EUM & $2(2.9)$ & $2(1.7)$ & $1(3.2)$ & \\
\hline Tumor size & & & & 0.548 \\
\hline$\leq 2 \mathrm{~cm}$ & $7(10.3)$ & $10(8.5)$ & $2(6.5)$ & \\
\hline$\sim \leq 5 \mathrm{~cm}$ & $35(51.5)$ & $56(47.5)$ & $10(32.2)$ & \\
\hline$\sim \leq 10 \mathrm{~cm}$ & $21(30.9)$ & $40(33.9)$ & $14(45.2)$ & \\
\hline$>10 \mathrm{~cm}$ & $5(7.4)$ & $12(10.2)$ & $5(16.1)$ & \\
\hline Multi-tumor & & & & 0.095 \\
\hline No & $68(100)$ & $115(97.5)$ & 29(93.5) & \\
\hline Yes & 0 & $3(2.5)$ & $2(6.5)$ & \\
\hline
\end{tabular}

Table 2 Patients' clinicopathologic parameters (Continued)

\begin{tabular}{cllll}
\hline Tstage $^{\text {a }}$ & & & & 0.059 \\
T2 & $5(7.4)$ & $11(9.3)$ & $2(6.5)$ & \\
T3 & $5(7.4)$ & $1(0.8)$ & $1(3.2)$ & \\
T4a & $51(75.0)$ & $101(85.6)$ & $23(74.2)$ & \\
T4b & $7(10.3)$ & $5(4.2)$ & $5(16.1)$ & \\
N stage & & & & 0.230 \\
N0 & $8(11.8)$ & $35(29.7)$ & $5(16.1)$ & \\
N1 & $13(19.1)$ & $16(13.6)$ & $7(22.6)$ & \\
N2 & $16(23.5)$ & $26(22.0)$ & $6(19.4)$ & \\
N3a & $18(26.5)$ & $21(17.8)$ & $8(25.8)$ & \\
N3b & $13(19.1)$ & $20(16.9)$ & $5(16.1)$ & \\
\hline
\end{tabular}

$B M I$ body mass index, NACT neoadjuvant chemotherapy, LND lymph node dissection, $L V I$ lymphovascular invasion, $E G J$ esophagogastric junction, $E$ esophagus, $U$ upper, $M$ middle

${ }^{a} 7$ th UICC/AJCC TNM classification for gastric cancer

including $8.8 \%(6 / 68)$ of the in situ group, $14.4 \%(17 /$ 118 ) of the ex situ group, and $32.3 \%(10 / 31)$ of the splenectomy group $(P=0.010)$. Of 466 harvested SHLNs, $11.4 \%(53 / 466)$ were metastatic, including $8.3 \%(10 / 121)$ in the in situ group, $11.8 \%(32 / 271)$ in the ex situ group, and $14.9 \%(11 / 74)$ in the splenectomy group $(P=0.349)$.

\section{Intraoperative and postoperative parameters}

Surgery-related parameters were compared among the three groups (Tables 3 and 4), and were found to differ significantly in the number of harvested SHLNs per patient $(P=0.047)$, length of postoperative hospital stay $(P=0.001)$, and blood loss volume $(P=0.027)$. Further paired comparisons revealed that the number of harvested SHLNs per patient was higher in the ex situ group than in the in situ group $(P=0.015)$. The length of postoperative hospital stay was significantly longer in the splenectomy group than in the other two groups (splenectomy vs in situ: $P=0.002$; splenectomy vs ex situ: $P<0.001$ ). The splenectomy group also had significantly greater blood loss volume than did the ex situ group $(P=0.007)$. The three groups did not significantly differ in total harvested lymph nodes per patient $(P=0.313)$ or operation duration $(P=0.695)$.

Postoperative complication rates were: in situ group: $17.6 \%$ (12/68); ex situ group: $12.7 \%$ (15/118); and splenectomy group: $35.5 \%(11 / 31 ; P=0.011)$; and were notably higher in the splenectomy group than in the ex situ group ( $P=0.005$; paired comparison). The three groups did not significantly differ in reoperation rate $(P=0.359)$ or postoperative mortality rate $(P=0.363)$.

\section{Survival outcomes}

As of April 26, 2016, median follow-up time was 33.2 months (range: 1-111 months). Median survival 
Table 3 Patients' intraoperative and postoperative parameters

\begin{tabular}{|c|c|c|c|c|}
\hline & In situ $(n=68)$ & Ex situ $(n=118)$ & Splenectomy $(n=31)$ & Pvalue \\
\hline No. of harvested SHLNs, median (range) & $1(1-4)$ & $2(1-7)$ & $2(1-7)$ & 0.047 \\
\hline No. of total harvested LNs, median (range) & $33(10-66)$ & $33(11-78)$ & $31(11-60)$ & 0.313 \\
\hline Postoperative hospital stay, days, (mean \pm standard deviation) & $16.41 \pm 3.06$ & $15.11 \pm 1.53$ & $23.26 \pm 4.74$ & 0.001 \\
\hline Blood loss volume, ml, (mean \pm standard deviation) & $211.62 \pm 53.43$ & $180.08 \pm 24.71$ & $262.90 \pm 78.09$ & 0.027 \\
\hline Operation duration, min, (mean \pm standard deviation) & $242.66 \pm 18.90$ & $244.24 \pm 13.66$ & $247.65 \pm 22.06$ & 0.695 \\
\hline Postoperative complication rate, $n$ (\%) & $12(17.6 \%)$ & $15(12.7 \%)$ & $11(35.5 \%)$ & 0.011 \\
\hline Bleeding, $n(\%)$ & $1(1.5 \%)$ & $3(2.5 \%)$ & $3(9.7 \%)$ & 0.153 \\
\hline Anastomotic leak, n (\%) & $3(4.4 \%)$ & $6(5.1 \%)$ & $5(16.1 \%)$ & 0.111 \\
\hline Pancreatic fistula, $n$ (\%) & $0(0.0 \%)$ & $0(0.0 \%)$ & $2(6.5 \%)$ & 0.019 \\
\hline Abdominal effusion, $n$ (\%) & $6(8.8 \%)$ & 7 (5.9\%) & $5(16.1 \%)$ & 0.183 \\
\hline Abdominal infection, $n$ (\%) & $6(8.8 \%)$ & $8(6.8 \%)$ & $7(22.6 \%)$ & 0.029 \\
\hline Pneumonia, n (\%) & $3(8.8 \%)$ & $1(0.8 \%)$ & $1(3.2 \%)$ & 0.269 \\
\hline Reoperation rate, $n$ (\%) & $3(4.4 \%)$ & $3(2.5 \%)$ & $2(6.5 \%)$ & 0.359 \\
\hline Mortality rate, $n$ (\%) & $0(0 \%)$ & $1(0.8 \%)$ & $1(3.2 \%)$ & 0.363 \\
\hline
\end{tabular}

SHLNs splenic hilar lymph nodes, LNs lymph nodes

times were: in situ group: 34.5 months, ex situ group: 71.1 months, and splenectomy group: 21.1 months; 5year overall survival rates were: in situ group: $46 \%$, ex situ group: $50 \%$, and splenectomy group: $23 \%$. The three groups were found to significantly differ by Kaplan-Meier survival analysis ( $\log$ rank test; $P=0.018$; Fig. 2 ), especially the splenectomy and ex situ groups $(P=0.005$; paired comparisons; Fig. 2).

Risk factors found in the univariate analysis included SHLN dissection method, operation duration, blood loss volume, postoperative complications, use of NACT, presence of multiple tumors, differentiation, tumor size, $\mathrm{T}$ stage, $\mathrm{N}$ stage, LVI, and range of gastrectomy (Table 5). All these factors were subjected to multivariate analysis, which found operation duration $(P=0.043)$, blood loss volume $(P=0.046)$, use of NACT $(P=0.005)$, and N stage $(P<0.001)$ to be independent prognostic factors for survival (Table 5).

Table 4 Pairwise comparisons of operative parameters and morbidity

\begin{tabular}{lccc}
\hline & \multicolumn{3}{l}{ Pvalue* } \\
\cline { 2 - 4 } & $\begin{array}{l}\text { In situ } \\
\text { vs splenectomy }\end{array}$ & $\begin{array}{l}\text { In situ } \\
\text { vs Ex situ }\end{array}$ & $\begin{array}{l}\text { Splenectomy vs } \\
\text { Ex situ }\end{array}$ \\
\hline No. of harvested SHLNs & 0.154 & 0.015 & 0.755 \\
Postoperative hospital stay & 0.002 & 0.832 & $<0.001$ \\
Blood loss volume & 0.058 & 0.388 & 0.007 \\
Postoperative & 0.047 & 0.239 & 0.005 \\
complication rate & & & \\
\hline
\end{tabular}

SHLNs splenic hilar lymph nodes, LNs lymph nodes *Bonferroni correction was carried out. $P<0.017$ (two-sided) was considered significant

\section{Discussion}

Although the incidence of gastric cancer has decreased worldwide, upper- and/or middle-third AGC has shown an increasing trend. As far as we know, the only way to cure gastric cancer is radical surgery, which includes gastrectomy and lymph node dissection. The currently recommended surgical procedure for advanced upperand/or middle-third gastric cancer is total gastrectomy with D2 lymph node dissection [7]. SHLNs are defined as group No.10 lymph nodes, which are included in D2 dissection. Reportedly, the incidence of SHLN metastasis in the upper- and/or middle-third AGC is 7.3-26\%, which was higher than in the lower-third gastric cancers [9-12, 15-17]. In our study, the incidence of metastasis of SHLNs was $15.2 \%$ (33/217), which was similar to previous reported studies, while the rates in the in situ, ex situ, and splenectomy groups were $8.8 \%(6 / 68), 14.4 \%$ $(17 / 118)$, and $32.3 \%(10 / 31)$, respectively $(P=0.010)$. Surgeons were inclined to use ex situ or splenectomy procedures for SHLNs suspected of having metastases, to perform dissections more effectively.

Optimal procedure for SHLN dissection has long been debated. Many previous studies have reported that splenectomy in this situation did not lead to longer survival $[12,18,19]$, and in fact might increase surgical complication and mortality rate. During splenectomy, the pancreas tail and spleen are mobilized, which often leads to pancreatic fistulae or abscess formation. Moreover, loss of the spleen and its effect on immune function might adversely affect the recovery process. In 2016, the JCOG 0110 study reported that prophylactic splenectomy should be avoided for both surgical safety and survival benefit in total gastrectomies for proximal 


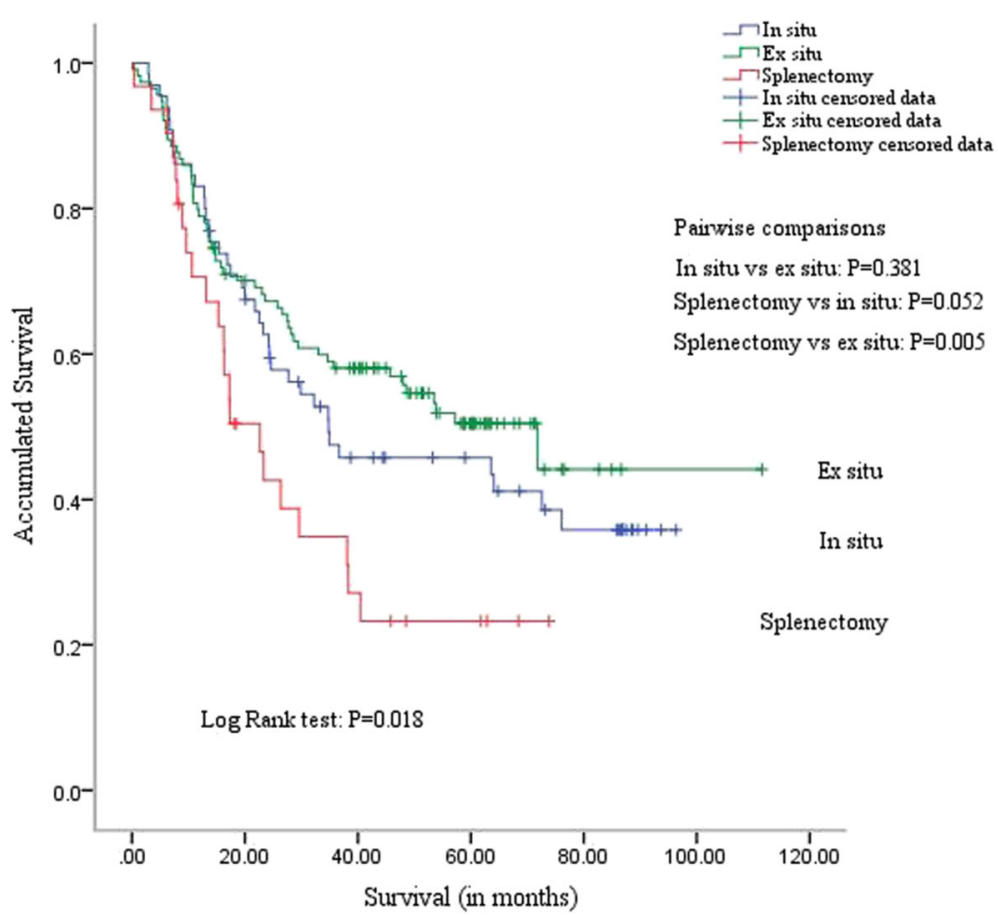

Fig. 2 Survival curves for three groups. The ex vivo, in vivo, and splenectomy groups significantly differed in survival $(P=0.018$, log-rank test). This difference was especially pronounced between the ex situ and splenectomy groups $(P=0.005, P<0.017)$. In the pairwise comparisons, Bonferroni modification was carried out. $P<0.017$ (two-sided) was considered significant

gastric cancers that do not invade the greater curvature [14]. Although patients whose cancers involved the greater curvature were not included in the JCOG 0110 study, it was the largest randomized clinical trial of splenectomy in gastric cancer, and demonstrated significant non-inferiority of spleen preservation for the first time. In our study, splenectomy reduced surgical safety and slowed the speed of postoperative recovery in terms of operative blood loss volume and postoperative hospital stay, compared with the spleen-sparing procedures. Our splenectomy group had longer average postoperative hospital stay, higher average blood loss volume, and a higher postoperative complication rate than the ex situ group, which was in accordance with earlier studies [19-21].

We found ex situ procedure was more effective for SHLN dissection than in situ splenic-preserving procedure and did not sacrifice surgical safety. Ex situ spleen-preserving procedure might improve the integrity of lymphadenectomy at the splenic hilum [22]. In the ex situ group, dissection of SHLNs was conducted under direct vision, and allowed surgeons to protect blood vessels and clear fatty tissues at the splenic hilum much more easily than in the in situ group, where dissection of SHLNs was very difficult and injury to spleen and blood vessels sometimes occurred. Therefore, although more time was required to mobilize the spleen and pancreas tail, the time needed to dissect SHLNs was significantly reduced. For this reason, operation duration was comparable between the in situ and ex situ groups. In our study, the ex situ procedure was more effective, and did not increase operation duration.

Interestingly, although Kaplan-Meier and log-rank analysis showed significant differences in survival among the three groups, Cox regression analysis of proportional hazards did not show SHLN dissection procedure to be an independent risk factor for survival. The significant difference shown in the Kaplan-Meier method might be caused by some other factors such as the imbalance of grouping in our study. The higher postoperative complication rate in the splenectomy group probably had adverse effects on survival, which is supported by earlier studies [23, 24].

Our study also had some limitations. First, it was a retrospective study, and selection bias was difficult to avoid. For instance, the percentages of patients who received NACT were much higher in the splenectomy and ex situ groups, probably because patients with later-stage disease were more likely to receive NACT. The choice of lymphadenectomy procedure was decided by surgeons, who usually chose patients with later-stage disease for ex situ or splenectomy procedures, as these methods seem to be more effective means to dissect the SHLNs. Similarly, more patients in the splenectomy or ex situ groups underwent 
Table 5 Univariate and multivariate analysis of prognostic factors

\begin{tabular}{|c|c|c|c|c|}
\hline & $\begin{array}{l}\text { Univariate HR } \\
(95 \% \mathrm{Cl})\end{array}$ & Pvalue & $\begin{array}{l}\text { Multivariate } \\
\text { HR }(95 \% \text { Cl) }\end{array}$ & Pvalue \\
\hline Gender & & 0.679 & & \\
\hline Male & 1 & & & \\
\hline Female & $0.909(0.580,1.427)$ & & & \\
\hline Age & & 0.254 & & \\
\hline$<60$ & 1 & & & \\
\hline$\geq 60$ & $1.238(0.857,1.788)$ & & & \\
\hline BMI & & 0.287 & & \\
\hline$<19$ & 1 & & & \\
\hline$\sim<25$ & $0.578(0.307,1.090)$ & 0.090 & & \\
\hline$\sim<30$ & $0.496(0.241,1.020)$ & 0.057 & & \\
\hline$\geq 30$ & $0.635(0.140,2.873)$ & 0.555 & & \\
\hline $\begin{array}{l}\text { Postoperative } \\
\text { hospital stay }\end{array}$ & $1.010(0.995,1.026)$ & 0.205 & & \\
\hline Reoperation & & 0.899 & & \\
\hline No & 1 & & & \\
\hline Yes & $1.067(0.393,2.896)$ & & & \\
\hline Location & & 0.100 & & \\
\hline EGJ & 1 & & & \\
\hline U/UM & $0.991(0.519,1.895)$ & 0.979 & & \\
\hline MU/M & $1.394(0.937,2.074)$ & 0.101 & & \\
\hline EUM & $2.908(1.048,8.071)$ & 0.040 & & \\
\hline $\begin{array}{l}\text { Degree } \\
\text { of LND }\end{array}$ & & 0.701 & & \\
\hline $\mathrm{D} 1+$ & 1 & & & \\
\hline D2 & $1.286(0.562,2.942)$ & 0.551 & & \\
\hline $\mathrm{D} 2+$ & $1.082(0.426,2.747)$ & 0.868 & & \\
\hline $\begin{array}{l}\text { SHLN } \\
\text { dissection } \\
\text { method }\end{array}$ & & 0.020 & & 0.061 \\
\hline In situ & 1 & & 1 & \\
\hline Ex situ & $0.822(0.541,1.249)$ & 0.369 & $0.804(0.510,1.269)$ & 0.349 \\
\hline Splenectomy & $1.671(0.983,2.840)$ & 0.058 & $1.522(0.865,2.678)$ & 0.145 \\
\hline $\begin{array}{l}\text { Operation } \\
\text { duration }\end{array}$ & $1.005(1.003,1.007)$ & $<0.001$ & $1.003(1.000,1.005)$ & 0.043 \\
\hline $\begin{array}{l}\text { Blood loss } \\
\text { volume }\end{array}$ & $1.001(1.001,1.002)$ & $<0.001$ & $1.001(1.000,1.002)$ & 0.046 \\
\hline $\begin{array}{l}\text { Postoperative } \\
\text { complications }\end{array}$ & & 0.036 & & 0.468 \\
\hline No & 1 & & 1 & \\
\hline Yes & $1.607(1.030,2.507)$ & & $1.210(0.723,2.027)$ & \\
\hline NACT & & 0.007 & & 0.005 \\
\hline No & 1 & & 1 & \\
\hline Yes & $1.689(1.157,2.466)$ & & $2.289(1.501,3.492)$ & \\
\hline Multi-tumor & & 0.011 & & 0.099 \\
\hline No & 1 & & 1 & \\
\hline Yes & $3.203(1.301,7.887)$ & & $2.402(0.849,6.800)$ & \\
\hline
\end{tabular}

Table 5 Univariate and multivariate analysis of prognostic factors (Continued)

\begin{tabular}{|c|c|c|c|c|}
\hline Differentiation & & 0.039 & & 0.319 \\
\hline Well & 1 & & 1 & \\
\hline Moderate & $1.200(0.512,2.816)$ & 0.674 & $1.155(0.474,2.818)$ & 0.751 \\
\hline Poor & $1.886(0.816,4.361)$ & 0.138 & $1.550(0.643,3.734)$ & 0.329 \\
\hline Tumor size & & 0.007 & & 0.135 \\
\hline$\leq 2 \mathrm{~cm}$ & 1 & & 1 & \\
\hline$\sim \leq 5 \mathrm{~cm}$ & $3.075(1.110,8.524)$ & 0.031 & $2.931(0.877,9.794)$ & 0.081 \\
\hline$\sim \leq 10 \mathrm{~cm}$ & $4.227(1.520,11.754)$ & 0.006 & $3.420(0.989,11.828)$ & 0.052 \\
\hline$>10 \mathrm{~cm}$ & $5.702(1.888,17.220)$ & 0.002 & $4.710(1.254,17.684)$ & 0.022 \\
\hline T stage ${ }^{a}$ & & 0.040 & & 0.572 \\
\hline $\begin{array}{l}\text { Serosa } \\
\text { negative }\end{array}$ & 1 & & 1 & \\
\hline T4a & $1.728(0.872,3.428)$ & 0.117 & $1.541(0.651,3.652)$ & 0.326 \\
\hline $\mathrm{T} 4 \mathrm{~b}$ & $3.036(1.275,7.227)$ & 0.012 & $1.645(0.604,4.482)$ & 0.330 \\
\hline N stage ${ }^{a}$ & & $<0.001$ & & $<0.001$ \\
\hline No & 1 & & 1 & \\
\hline N1 & $1.482(0.755,2.908)$ & 0.253 & $1.398(0.696,2.808)$ & 0.347 \\
\hline N2 & $1.937(1.308,3.616)$ & 0.038 & $1.836(0.933,3.609)$ & 0.078 \\
\hline N3a & $1.714(0.904,3.250)$ & 0.099 & $1.958(0.988,3.883)$ & 0.054 \\
\hline N3b & $5.441(2.950,10.033)$ & $<0.001$ & $6.327(3.181,12.582)$ & $<0.001$ \\
\hline LVI & & 0.004 & & 0.334 \\
\hline No & 1 & & 1 & \\
\hline Yes & $1.727(1.182,2.523)$ & & $1.299(0.764,2.209)$ & \\
\hline Gastrectomy & & 0.001 & & 0.203 \\
\hline Proximal & 1 & & 1 & \\
\hline Total & $2.098(1.336,3.224)$ & & $1.368(0.844,2.218)$ & \\
\hline
\end{tabular}

$B M I$ body mass index, $E G J$ esophagogastric junction, $E$ esophagus, $U$ upper, $M$ middle, LND lymph node dissection, SHLN splenic hilar lymph node, NACT neoadjuvant chemotherapy, LVI lymphovascular invasion

${ }^{a} 7$ th UICC/AJCC TNM classification for gastric cancer

total gastrectomies, which are more suitable for patients with later-stage disease. The three groups did not significantly differ with regard to other clinicopathologic parameters. Second, as the sample size in the splenectomy group was much smaller than that in the other two groups, a type II error might have occurred.

\section{Conclusions}

Ex situ SHLN dissections were safer than splenectomies. Compared with in situ procedures, ex situ procedures apparently dissected SHLNs more effectively. Although the survival in these three groups significantly differed in Kaplan-Meier analysis, SHLN dissection method was not an independent risk factor for survival. Multicenter, largescaled, randomized controlled trials are needed to clarify the optimal splenic hilar lymphadenectomy procedure. 


\section{Abbreviations}

AGC: Advanced gastric cancer; BMI: Body mass index; JCOG: Japanese Clinical Oncology Group; LND: Lymph node dissection; LVI: Lymphovascular invasion; NACT: Neoadjuvant chemotherapy; SHLN: Splenic hilar lymph node (No. 10 lymph node)

\section{Acknowledgments}

We thank the staff members at the database center at Peking University Cancer Hospital and Institute for assistance with the data search and project management. We also thank Ying Ji from Peking University 3rd Affiliated Hospital for help in preparing this manuscript.

\section{Funding}

Not applicable.

\section{Availability of data and materials}

All relevant materials are provided in the manuscript.

\section{Authors' contributions}

$J J$ designed this study and was in charge of its coordination. XJ, TF, ZB, JZ, $X W, X Z, Z J, B F$, and $Y Z$ participated in the clinical data collection. $X J$ and TF conducted the statistical analysis. $X J$ drafted the manuscript. $X J$ and TF are joint first authors. All authors read and approved the final manuscript.

\section{Authors' information}

1. Jia-Fu Ji: Director of Peking University Cancer Hospital, Chief Expert of Gastric Cancer Collaborative Group of China, Chairman of the Gastric Cancer Association of Chinese Anti-Cancer Association, Director of International Cooperation Department of China Medical Association, Guest Professor of Surgery of the Gastroenterologists and Oncologists Academy in Europe, Member of American College of Surgeons, Vice Chairman of Expert Committee of Nutritional Therapy for Cancer, Chairman of Tumor Examination Committee of Cross-straits Medicine Exchange Association, Member of Asian Surgical Association, Member of European Society for Clinical Nutrition and Metabolism, evaluation expert of National Science Foundation and 863 Major Projects. 2. Department of Gastrointestinal Surgery of Peking University Cancer Hospital and Institute: Performing standard radical gastrointestinal surgery and multidisciplinary treatment, learning center of multidisciplinary treatment supported by the Chinese Anticancer Association and the Chinese Medical Association, IASGO CME Center.

\section{Competing interests}

The authors declare that they have no competing interests.

\section{Consent for publication}

Not applicable.

\section{Ethics approval and consent to participate}

This study was performed in accordance with the Declaration of Helsinki, and was approved by the Ethics Committee of Peking University Cancer Hospital (Reference No. 2006021). Informed consent was obtained from each patient.

\section{Received: 24 June 2016 Accepted: 26 September 2016}

\section{Published online: 03 October 2016}

\section{References}

1. Global Burden of Disease Cancer C, Fitzmaurice C, Dicker D, Pain A, Hamavid H, Moradi-Lakeh M, et al. The global burden of cancer 2013. JAMA Oncol. 2015;1(4):505-27.

2. Ferlay J, Soerjomataram I, Dikshit R, Eser S, Mathers C, Rebelo M, et al. Cancer incidence and mortality worldwide: sources, methods and major patterns in GLOBOCAN 2012. Int J Cancer. 2015;136(5):E359-86.

3. Songun I, Putter H, Kranenbarg EM, Sasako M, van de Velde CJ. Surgical treatment of gastric cancer: 15-year follow-up results of the randomised nationwide Dutch D1D2 trial. Lancet Oncol. 2010;11(5):439-49.

4. Sano T, Sasako M, Yamamoto S, Nashimoto A, Kurita A, Hiratsuka M, et al. Gastric cancer surgery: morbidity and mortality results from a prospective randomized controlled trial comparing D2 and extended para-aortic lymphadenectomy-Japan Clinical Oncology Group study 9501. J Clin Oncol. 2004;22(14):2767-73.

5. Van Cutsem E, Sagaert X, Topal B, Haustermans K, Prenen H. Gastric cancer. Lancet. 2016; doi:10.1016/S0140-6736(16)30354-3.
6. Dubecz A, Solymosi N, Stadlhuber RJ, Schweigert M, Stein HJ, Peters JH. Does the incidence of adenocarcinoma of the esophagus and gastric cardia continue to rise in the twenty-first century?-a SEER database analysis. J Gastrointest Surg. 2013; doi:10.1007/s11605-013-2345-8.

7. Japanese Gastric Cancer A. Japanese gastric cancer treatment guidelines 2010 (ver. 3). Gastric Cancer. 2011;14(2):113-23.

8. Japanese Gastric Cancer A. Japanese classification of gastric carcinoma: 3rd English edition. Gastric Cancer. 2011;14(2):101-12.

9. Okajima K, Isozaki H. Splenectomy for treatment of gastric cancer: Japanese experience. World J Surg. 1995;19(4):537-40.

10. Ikeguchi M, Kaibara N. Lymph node metastasis at the splenic hilum in proximal gastric cancer. Am Surg. 2004;70(7):645-8

11. Sasada S, Ninomiya M, Nishizaki M, Harano M, Ojima Y, Matsukawa $H$, et al. Frequency of lymph node metastasis to the splenic hilus and effect of splenectomy in proximal gastric cancer. Anticancer Res. 2009:29(8):3347-51.

12. Monig SP, Collet PH, Baldus SE, Schmackpfeffer K, Schroder W, Thiele J, et al. Splenectomy in proximal gastric cancer: frequency of lymph node metastasis to the splenic hilus. J Surg Oncol. 2001;76(2):89-92.

13. Kosuga T, Ichikawa D, Okamoto K, Komatsu S, Shiozaki A, Fujiwara H, et al. Survival benefits from splenic hilar lymph node dissection by splenectomy in gastric cancer patients: relative comparison of the benefits in subgroups of patients. Gastric Cancer. 2011;14(2):172-7.

14. Sano T, Sasako M, Mizusawa J, Yamamoto S, Katai H, Yoshikawa T, et al. Randomized controlled trial to evaluate splenectomy in total gastrectomy for proximal gastric carcinoma. Ann Surg. 2016; doi:10.1097/SLA. 0000000000001814.

15. Kim JH, Park SS, Kim J, Boo YJ, Kim SJ, Mok YJ, et al. Surgical outcomes for gastric cancer in the upper third of the stomach. World J Surg. 2006;30(10): 1870-6. discussion 7-8.

16. Sun Z, Wang Q, Yu X, Ou C, Yao L, Liu K, et al. Risk factors associated with splenic hilar lymph node metastasis in patients with advanced gastric cancer in northwest China. Int J Clin Exp Med. 2015;8(11):21358-64.

17. Chen $X L$, Yang $K$, Zhang WH, Chen XZ, Zhang B, Chen ZX, et al. Metastasis, risk factors and prognostic significance of splenic hilar lymph nodes in gastric adenocarcinoma. PLoS One. 2014;9(6):e99650.

18. Yu W, Choi GS, Chung HY. Randomized clinical trial of splenectomy versus splenic preservation in patients with proximal gastric cancer. Br J Surg. 2006 93(5):559-63.

19. Weitz J, Jaques DP, Brennan M, Karpeh M. Association of splenectomy with postoperative complications in patients with proximal gastric and gastroesophageal junction cancer. Ann Surg Oncol. 2004;11(7):682-9.

20. Csendes A, Burdiles P, Rojas J, Braghetto I, Diaz JC, Maluenda F. A prospective randomized study comparing D2 total gastrectomy versus D2 total gastrectomy plus splenectomy in 187 patients with gastric carcinoma. Surgery. 2002;131(4):401-7

21. Hartgrink $\mathrm{HH}$, van de Velde CI. Status of extended lymph node dissection: locoregional control is the only way to survive gastric cancer. J Surg Oncol. 2005:90(3):153-65

22. Yang K, Lu ZH, Zhang WH, Liu K, Chen XZ, Chen XL, et al. Comparisons between different procedures of no. 10 lymphadenectomy for gastric cancer patients with total gastrectomy. Medicine (Baltimore). 2015;94(33):e1305.

23. Degiuli M, Sasako M, Ponzetto A, Allone T, Soldati T, Calgaro M, et al. Extended lymph node dissection for gastric cancer: results of a prospective, multi-centre analysis of morbidity and mortality in 118 consecutive cases. Eur J Surg Oncol. 1997;23(4):310-4.

24. Fatouros M, Roukos DH, Lorenz M, Arampatzis I, Hottentrott C, Encke A, et al. Impact of spleen preservation in patients with gastric cancer. Anticancer Res. 2005;25(4):3023-30. 